\title{
Traditional and non-traditional anticoagulation management during extracorporeal membrane oxygenation
}

\author{
Andreas Koster ${ }^{1}$, Edis Ljajikj ${ }^{2}$, David Faraoni ${ }^{3}$ \\ ${ }^{1}$ Institute of Anesthesiology, ${ }^{2}$ Department of Thoracic and Cardiovascular Surgery, Heart and Diabetes Center NRW, Ruhr-University Bochum, \\ Bad Oeynhausen, Germany; ${ }^{3}$ Department of Anesthesia and Pain Medicine, Hospital for Sick Children, University of Toronto, Toronto, Canada \\ Correspondence to: Andreas Koster, MD. Institute of Anesthesiology, Heart and Diabetes Center NRW, Georgstr. 11, D-32545 Bad Oeynhausen, \\ Germany. Email: akoster@hdz-nrw.de.
}

\begin{abstract}
Unfractionated heparin (UFH) is the anticoagulant of choice during extracorporeal membrane oxygenation (ECMO) support. Despite its favorable pharmacologic properties, management of heparin anticoagulation during ECMO remains a major challenge. To date, little is known about the optimal monitoring strategy or the heparin dose offering the best safety/efficacy profile. Therefore, it remains unclear if the heparin dose should be adapted to target a specific "clotting time" [e.g., activated clotting time (ACT) or activated partial thromboplastin time (aPTT)] or a heparin concentration, measured by coagulation factor anti-Xa assay. In addition, no study has compared the relevance of modern viscoelastic coagulation tests over the single value of a clotting time or heparin concentration value. Although guidelines for anticoagulation during ECMO support have been published, the absence of evidence limits the quality of the recommendations provided, which explains the major intra- and inter-institutional variability observed. Large prospective multicenter trials are urgently needed to investigate the optimal anticoagulation management strategy during ECMO support.
\end{abstract}

Keywords: Bleeding; thrombosis; risk

Submitted May 03, 2018. Accepted for publication Jul 13, 2018.

doi: $10.21037 /$ acs.2018.07.03

View this article at: http://dx.doi.org/10.21037/acs.2018.07.03

\section{Introduction}

Children and adults supported with extracorporeal membrane oxygenation (ECMO) frequently experience bleeding and thrombotic complications. In a review of the 2014 data from the Extracorporeal Life Support Organization (ELSO), the incidence of bleeding at the surgical or cannula insertion sites ranged between $10 \%$ and $30 \%$ and central nervous system hemorrhage from $2.2 \%$ to $6 \%$. The incidence of oxygenator thrombosis ranged from $7 \%$ to $13 \%$ and central nervous system infarction from $2 \%$ to $4.4 \%$ (1). In a recent meta-analysis of observational studies including 1,496 patients supported with venoarterial (VA)-ECMO, the incidence of major bleeding ranged from $13 \%$ to $50 \%$ and was correlated to the heparin monitoring strategy used (Table 1) (2). The main contributors to hemorrhagic complications were surgical re-exploration, intracranial hemorrhage and bleeding at the site of cannula insertion. The incidence of thrombotic complications ranged from $3 \%$ to $12 \%$ and was also correlated with the applied heparin monitoring strategy. The thrombotic events predominately included limb ischemia, circuitrelated clotting and stroke (Table 1). Another meta-analysis including 1,763 patients supported by ECMO showed that bleeding and thrombotic events predominately contributed to complications during ECMO support (3). Bleeding complications are considered to be major contributors of morbidity and mortality during ECMO support (1). In a large two-center study in 149 ECMO runs, bleeding complications were associated with an increased (adjusted hazard ratio 2.17; CI: 1.07-4.41, $\mathrm{P}=0.03$ ) likelihood of mortality (4).

The etiology of bleeding and thrombosis during ECMO support is complex and multifactorial. The contact of blood with the non-endothelial surfaces, even with the use of biocompatible materials, leads to the 


$\begin{aligned} & \text { Table } 1 \text { Meta-analysis of bleeding and thrombotic complications } \\
& \text { during VA-ECMO with heparin depending on coagulation tests (2) }\end{aligned}$
\begin{tabular}{lll}
\hline Assay range & Major bleeding (\%) & Thrombosis (\%) \\
\hline ACT $<180$ seconds & 13 & 12 \\
ACT $>180$ seconds & 28 & 9 \\
aPTT & 50 & 3 \\
\hline
\end{tabular}

VA-ECMO, venoarterial extracorporeal membrane oxygenation; ACT, activated clotting time; aPTT, activated partial thromboplastin time. activation of the coagulation cascade and progressive consumption of coagulation factors and platelets, in addition to excessive fibrinolytic activation $(5,6)$. In these critically-ill patients, impaired organ function, infection/ sepsis and inflammation may further contribute to the disturbance of the inflammation/coagulation system $(5,6)$. Convincing data suggest that ECMO support leads to an acquired type II von Willebrand (vW) syndrome (7); the high velocity ECMO blood flow leads to an uncoiling of the high molecular weight multimers of the $\mathrm{vW}$ factor, rendering this domain susceptible to proteolytic cleavage by the metalloproteinase ADAMTS-13 (7). Since high molecular weight multimers play a pivotal role in the endothelium/platelet interaction, primary hemostasis is severely disturbed. In addition, ECMO support, and sometimes prolonged heparin anticoagulation, appear to be associated with an increased risk of heparin induced thrombocytopenia (HIT). In selected patients, the HIT antibodies activate platelets and thrombin generation, promoting thromboembolism (8). Last, but not least, ECMO will require adequate anticoagulation in order to avoid fibrin deposition and potentially lethal clot formation. "The challenge in anticoagulation is to reduce the incidence of thrombotic events without increasing the risk of bleeding complications". Unfortunately, even with optimal dosage and monitoring, bleeding during anticoagulation remains a major source of morbidity and mortality (9). Although this principally applies for all anticoagulants and clinical contexts, it perfectly reflects the challenge of anticoagulation during ECMO support.

In this review, we will focus on traditional (i.e., heparin) and non-traditional [i.e., direct thrombin inhibitors (DTIs)] anticoagulation management strategies in patients supported by ECMO. We will review evidence regarding anticoagulation with unfractionated heparin (UFH) as well as its monitoring. We will also briefly address alternatives to UFH, their indications, doses and monitoring.

\section{Monitoring of anticoagulation with UFH}

UFHs are considered to be the gold standard for parenteral anticoagulation (10). UFH is easily titratable and reversible through the administration of protamine. The doses of UFH administered vary widely and are adjusted until a targeted anticoagulation effect is achieved. Unfortunately, management of UFH anticoagulation largely depends on the coagulation assay used and important inter- and intra-individual variabilities have been reported. To date, two management strategies are routinely used. The "clotting time" approach, based on either standard laboratory testing [e.g., activated partial thromboplastin time (aPTT)] or the whole blood pointof-care activated clotting time (ACT), or a "chromogenic" heparin concentration approach, based on anti-factor Xa activity measurement (11). The absence of standardization in ECMO patients has been highlighted in two recent surveys in pediatric and adult patients (Table 2) $(12,13)$.

\section{"Clotting time" based heparin monitoring with ACT or aPTT}

The aPTT and ACT are assays measuring the time between ex-vivo contact activation the of coagulation cascade and the formation of a fibrin/thrombin "gel" into the tube. In the "clotting time"-based anticoagulation approach, UFH dosing will be adjusted to achieve a targeted prolongation of the clotting time (e.g., 1.5 to $2.5 \times$ baseline value for the aPTT). In addition to pre-analytic and analytic variabilities, none of those assays are specific for unfractionated heparin (UFH) anticoagulation and will therefore be influenced by the presence of an underlying coagulopathy (i.e., biologic variability). In patients with inherited (e.g., hemophilia, $\mathrm{vW}$ disease type 3) or acquired (e.g., vitamin $\mathrm{K}$ antagonist anticoagulation, liver failure, dilutional coagulopathy) coagulation disorders, lower UFH concentrations/dosages will be required to prolong the clotting time within the targeted ranges. However, other situations such as hyperfibrinogenemia, antithrombin (AT) deficiency and thrombocytosis may call for increased, sometimes excessive, dosages of UFH, a condition called "heparin resistance" (14).

Although the aPTT and ACT reflect the same pathway of the coagulation cascade, a number of differences have been noted. The ACT was originally designed for "point 


\begin{tabular}{|c|c|c|c|c|c|}
\hline \multicolumn{6}{|c|}{ Pediatric network survey (11) } \\
\hline No. Inst. (\%) & 97 & 94 & 65 & 82 & n.i. \\
\hline Range (\%) & $180-200$ s [38] & n.i. & 0.3-0.7 IE/mL [65] & $30-100 \%[60]$ & n.i. \\
\hline No. Inst. (\%) & 30 & 51 & & 50 & 98 \\
\hline $\begin{array}{l}\text { Predominant range } \\
\text { (Inst. \%) }\end{array}$ & $\begin{array}{l}160-200 \mathrm{~s} \\
\text { (approx. 50) }\end{array}$ & $\begin{array}{l}40-60 \text { s (approx. 50) } \\
60-80 \text { s (approx. 50) }\end{array}$ & 0.3-0.7 IE/mL [65] & $\begin{array}{l}50-100 \% \\
\text { (approx. 40) }\end{array}$ & $<5010^{3} / \mu \mathrm{L}[50]$ \\
\hline
\end{tabular}

of care" monitoring of high-dose anticoagulation during cardiopulmonary bypass (CPB). Although modifications have been made to improve accuracy when used to monitor lower UFH concentrations (e.g., low-range ACT cartridges), the target range of these tests is still significantly higher than during ECMO support. The reproducibility of those tests is limited by the variability between assays with regard to the activator used (e.g., celite, kaolin, celite and phospholipids) and the technique used for clot detection $(15,16)$. Moreover, intervariability can be observed, as the precision of the technique is operator-dependent (16). As ACT is performed on whole blood, the test is influenced by a variety of conditions, including temperature, hematocrit and the platelet count $(15,16)$.

In contrast, the aPTT has been designed to monitor UFH in prophylactic and therapeutic ranges $(10,17)$. The test is usually performed in the laboratory, increasing the reliability of test results. Nevertheless, different laboratory methods can be used which limit the inter-assay/interinstitution reproducibility $(11,18)$. The aPTT, measured in platelet poor plasma, is not influenced by the platelet count or hematocrit. This condition might explain the fact that the aPTT, when compared ACT assays, better correlates to heparin concentrations during ECMO support (19). ACT and aPTT during ECMO support has been compared in a large number of investigations. However, when viewing the large variety of tests used, it is not surprising that results are conflicting. While in some investigations a good correlation between both tests was found, a weak correlation was reported in some other investigations (19-21).

In a meta-analysis of observational studies in patients supported with VA-ECMO, the incidences of bleeding and thrombotic complications were compared based on anticoagulation monitoring used (e.g., ACT vs. aPTT monitoring) (Table 1). Even though the validity of this subanalysis is limited by the retrospective nature of the analysis and the heterogeneity between anticoagulation protocols, UFH anticoagulation based on ACT measurements seemed to be associated with fewer major bleeding complications (2). The authors also reported an association between lower ACT targets and an increased incidence of thrombotic complications. On the other hand, anticoagulation monitoring based on aPTT was associated with more major bleeding complications, but less thrombotic events (Table 1).

\section{Heparin-concentration based monitoring with anti-Xa assays}

In chromogenic anti-factor Xa assays, a coagulation factor $\mathrm{X}$ substrate with a linked chromophore is added to the patient's plasma. Activated factor $\mathrm{X}$ is then added and cleaves the chromophore compound. The amount of chromophore released is measured by means of spectroscopy. As defined amounts of factor $\mathrm{Xa}$ are added, the results can be correlated to determine factor $\mathrm{Xa}$ inhibition by plasmatic heparin $(11,18)$. Therefore, chromogenic anti-Xa assays do not reflect the overall condition of the coagulation system, but the heparin concentration/activity in the patient's blood. Notably, in some tests, AT is added to the assays, while in others this is not the case. Therefore, in assays with supplemented AT, the result of the in vitro test might not reflect the patient's in vivo heparin activity. The established target anti-factor $\mathrm{Xa}$ value for therapeutic anticoagulation is $0.3-0.7 \mathrm{IU} / \mathrm{mL}$ (17). 
In a large prospective single center investigation in 109 patients, anti-factor Xa levels were analyzed when targeting the ACT value at 180-220 seconds, which was the basis for the adjustment of the heparin infusion. The anti$\mathrm{Xa}$ target was $0.2-0.4 \mathrm{IU} / \mathrm{mL}$. This study showed that even when using this low anti-factor Xa target, a large number of anti-Xa values were below the target range (22). Moreover, the correlation between both assays was poor. The authors concluded that in ECMO patients, concomitant measurement of ACT and anti-factor Xa values is irrelevant.

In a large retrospective analysis of more than 22,000 adult medical patients, anti-Xa-based heparin management significantly reduced transfusions when compared to an aPTT-based strategy (23). In a retrospective study of $100 \mathrm{ECMO}$ and non-ECMO children, therapeutic anti-Xa levels were associated with a $66 \%$ reduction in the thrombosis rate, while $11 \%$ of patients experienced bleeding, particularly when aPTT values were excessive (24). Obviously, these results cannot be simply translated to the high-risk group of patients on ECMO support, especially in the instance where ECMO support is initiated after a major surgery. In a retrospective analysis of 62 pediatric patients on ECMO, anti-Xa concentrations $<1.3 \mathrm{U} / \mathrm{mL}$ were associated with an increased need for oxygenator change, irrespective of the ACT results (25). In 22 adult patients on ECMO support, anti-Xa levels were inversely correlated with the occurrence of deep vein thrombosis (26). A large, retrospective single-center investigation of 365 pediatric ECMO patients showed a reduction in the incidence of bleeding and transfusion requirements, as well as decreased numbers of circuit replacement due to clotting after implementing an anti-factor Xa target of 0.3-07 IU/mL, daily AT level control and AT replacement, when heparin demand exceeded $60 \mathrm{U} / \mathrm{kg} / \mathrm{h}$ (27). Additionally, another large, retrospective single-center investigation in pediatric patients compared an ACT-based protocol (target 160-200 seconds) with an anti-factor Xa-based protocol (target $0.5-0.7 \mathrm{IU} / \mathrm{mL}$ ) (28). The analysis included 152 ECMO runs in the ACT group and $122 \mathrm{ECMO}$ runs in the anti-factor $\mathrm{Xa}$ group. In this investigation, an antifactor Xa-based heparin management was associated with an improvement in survival, a decrease in hemorrhagic complications, transfusions and improved circuit patency. Notably, however, this analysis covered a period of 10 years and changes in anticoagulation were also accompanied by changes in ECMO circuit technology, assumedly introducing heterogeneity. Moreover, although the groups appeared to be comparable, no risk-adjustment between groups was performed. The results concerning standard AT replacement are conflicting as well. A larger retrospective study in 162 neonatal ECMO patients showed a reduction of thrombotic events after standard implementation of AT replacement, without an increase in hemorrhagic complications (29). However, results could not be confirmed in other smaller retrospective single-center investigations $(30,31)$.

In summary, there is increasing evidence coming from observational studies, particularly performed in pediatric ECMO patients, suggesting that an anti-factor Xa-based heparin management could be associated with improved results.

\section{Viscoelastic tests and anticoagulation during ECMO support}

Over the past decade, viscoelastic tests (e.g., ROTEM or TEG) have been increasingly used in different clinical conditions to guide the administration of blood products in the context of excessive bleeding $(32,33)$. Besides monitoring anticoagulation, viscoelastic tests provide a view of clot formation dynamics, clot strength and clot lysis. The synoptic analysis of different channels with different activators of the coagulation system and heparinase, which specifically inhibits UFH, provides a deeper insight into the complex anticoagulation-coagulation system. Viscoelastic tests have only been the subject of a few ECMO studies, however, it is conceivable that the balance between therapeutic anticoagulation and the assessment of global clot formation and stability could improve coagulationanticoagulation management in children and adults supported by ECMO (34). Although these tests appear to provide a real picture of the complex anticoagulation and coagulation system, important disturbances of primary hemostasis, such as the acquired $\mathrm{vW}$ deficiency, would not be reflected in these assays.

\section{Alternatives to UFH: argatroban and bivalirudin anticoagulation during ECMO support}

The DTIs, argatroban and bivalirudin, have been used as alternative anticoagulation strategies during ECMO support (35). Additionally, both drugs have been used within the perioperative setting of cardiac surgery (35). Argatroban is approved in the United States and most European countries for anticoagulation in patients with, or at risk of, HIT, while bivalirudin is used "off-label" for this indication. The pharmacokinetic characteristics of each 


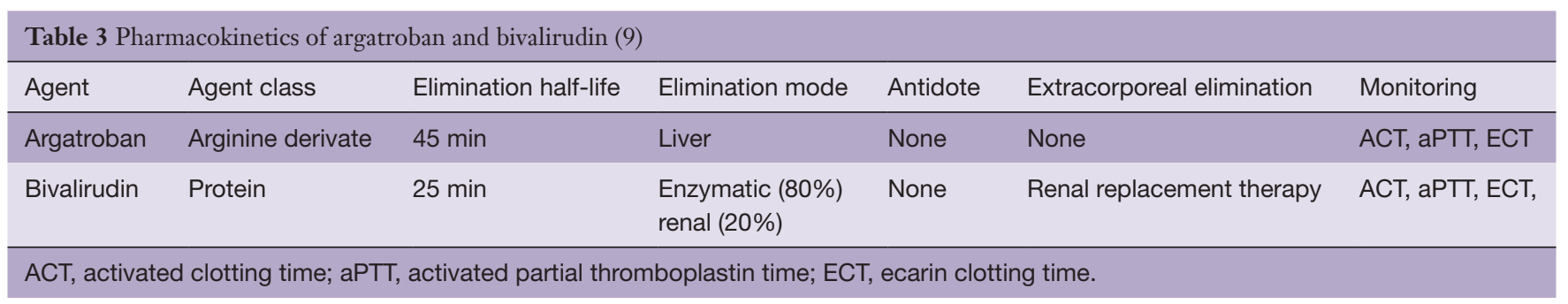

drug are provided in Table 3 (10).

Experience and data regarding the use of DTIs for anticoagulation in ECMO patients is essentially limited to cases of HIT (35-37). Only a few studies have compared DTIs anticoagulation to standard UFH anticoagulation $(38,39)$. Dosing is usually adapted to reach target aPTT values comparable to the targeted values recommended for UFH anticoagulation during ECMO support. As both DTIs interfere with the prothrombin time (40), the results of this test cannot be used to guide therapy with plasmatic coagulation factors. The dosing for argatroban during ECMO support ranges within $0.2-0.5 \mu \mathrm{g} / \mathrm{kg} / \mathrm{min}$, while the bivalirudin dose varies between 0.025 to $0.05 \mathrm{mg} / \mathrm{kg} / \mathrm{min}$. Viewing all available data for both agents, the anticoagulant effect during ECMO support is predictable and stable. Moreover, the clinical results with regard to ECMO system patency and bleeding complications appear to be comparable to UFH anticoagulation.

As both agents present comparable pharmacokinetic characteristics, one drug cannot be recommended over the other. However, because of the absence of reversal agent, bivalirudin should be preferred over argatroban in patients with a high bleeding risk, as renal replacement therapy provides an effective tool for rapid elimination of the drug when urgent surgery has to be performed (35). Based on the limited literature and the risks associated with their utilization, DTI anticoagulation should be limited to cases of HIT or in the instance where contraindications to UFH are present.

\section{Intraoperative anticoagulation during surgery with ECMO support}

ECMO systems are being increasingly used intraoperatively as a replacement of $\mathrm{CPB}$, or as a bridge to transplantation. Therefore, an increasing number of patients will undergo surgical procedures while on ECMO [e.g., ventricular assist device (VAD) implantation or transplantation] $(41,42)$. The closed, small, often biocompatible-coated circuit reduces hemodilution and allows reduced or minimal systemic anticoagulation. Depending on the procedure, the usual target ACT range is 160-220 seconds (35). This significant dose reduction appears to be particularly important in patients with HIT, as no reversal for the current alternative anticoagulants is available. A more recent larger series, using low-dose bivalirudin anticoagulation during left ventricular assist device (LVAD) implantation, revealed promising results (43). However, the use of argatroban during a comparable procedure was associated with severe bleeding and therefore should be discouraged (44).

\section{Comments}

Patients on ECMO support represent a rather heterogeneous group, ranging from small neonates with immature coagulation systems, to old adults and patients with isolated lung replacement therapy with venovenous (VV)-ECMO. Additionally, the presence of complex patients receiving hybrid approaches with both VV- and VA-ECMO, patients needing ECMO within the setting of medical treatment and patients receiving ECMO after major surgery or trauma, all make sub-group analysis and interpretation of results challenging. Bleeding and thrombotic complications are major contributors to morbidity and mortality during ECMO. However, all these conditions may impact the risk for bleeding and thrombosis.

Although alternatives to classic UFH anticoagulation agents have been assessed, data are limited and anticoagulation with UFH remains the gold standard. Unfortunately, the optimal anticoagulation strategy with intravenous UFH during ECMO support remains a subject of uncertainty and intense debate. Current ELSO recommendations are more descriptive rather than practical (45). In this regard, the special situation during ECMO support reflects the overall dilemma in therapeutic anticoagulation with UFH (46). All methods for monitoring and directing UFH 
anticoagulation (e.g., ACT, aPTT, and anti-factor Xa levels) have their advantages and disadvantages, but it is clear that the ACT and aPTT are wrought with problems. Both tests are affected by many biologic factors unrelated to heparin concentration. Guiding UFH anticoagulation only on the basis of such tests has been declared an "unsafe practice" (47). In contrast, maintaining target anti-Xa concentrations most likely reduces the incidence of thrombotic events. Results from pediatric patients are promising, and discounting how far these results can be translated to the adult wards, the fundamental limitations of the observational and single-center nature of these studies have to be clearly considered. Moreover, in ECMO patients with an impaired overall coagulation system, particularly after major surgery, such a strategy may be associated with an increased risk of bleeding (23).

When viewing the underlying complexity of balancing anticoagulation and bleeding risk in ECMO patients, one could argue that a combination of anti-Xa assays and viscoelastic tests could improve coagulation assessment and presumably reduce the bleeding risk. Large, controlled studies are needed to validate this hypothesis.

In summary, the lack of evidence provided by adequately powered, prospective, randomized, multicenter trials are the key limitation for defining the optimal anticoagulationcoagulation management strategy in ECMO patients. Noting that UFH-based drugs are established and inexpensive, much like the laboratory assays, no new studies can be expected to be performed from these sites. This point is made particularly clear when the field of interest is restricted to a very special condition, such as ECMO support. Seeing the obvious need for reliable data, alternative strategies have to be developed. One possible strategy might be that such studies are funded by independent national or international organizations, such as the Food and Drug Administration (FDA) or European Medicines Agency (EMA), and performed by independent organizations such as the ELSO. A wider discussion in this regard would be highly appreciated.

\section{Acknowledgements}

None.

\section{Footnote}

Conflicts of Interest: The authors have no conflicts of interest to declare.

\section{References}

1. Murphy DA, Hockings LE, Andrews RK, et al. Extracorporeal membrane oxygenation-hemostatic complications. Transfus Med Rev 2015;29:90-101.

2. Sy E, Sklar MC, Lequier L, et al. Anticoagulation practices and the prevalence of major bleeding, thromboembolic events, and mortality in venoarterial extracorporeal membrane oxygenation: A systematic review and metaanalysis. J Crit Care 2017;39:87-96

3. Zangrillo A, Landoni G, Biondi-Zoccai G, et al. A metaanalysis of complications and mortality of extracorporeal membrane oxygenation. Crit Care Resusc 2013;15:172-8.

4. Aubron C, DePuydt J, Belon F, et al. Predictive factors of bleeding events in adults undergoing extracorporeal membrane oxygenation. Ann Intensive Care 2016;6:97.

5. Esper SA, Levy JH, Waters JH, et al. Extracorporeal membrane oxygenation in the adult: a review of anticoagulation monitoring and transfusion. Anesth Analg 2014;118:731-43.

6. Andrews J, Winkler AM. Challenges with Navigating the Precarious Hemostatic Balance during Extracorporeal Life Support: Implications for Coagulation and Transfusion Management. Transfus Med Rev 2016;30:223-9.

7. Heilmann C, Geisen U, Beyersdorf F, et al. Acquired von Willebrand syndrome in patients with extracorporeal life support (ECLS). Intensive Care Med 2012;38:62-8.

8. Laverdure F, Louvain-Quintard V, Kortchinsky T, et al. PF4-heparin antibodies during ECMO: incidence, course, and outcomes. Intensive Care Med 2016;42:1082-3.

9. Crowther M, Crowther M. Management of bleeding in patients on antithrombotics: maintaining the balance between thrombosis and hemorrhage. Expert Rev Hematol 2009;2:357-60.

10. Garcia DA, Baglin TP, Weitz JI, et al. Parenteral anticoagulants: Antithrombotic Therapy and Prevention of Thrombosis, 9th ed: American College of Chest Physicians Evidence-Based Clinical Practice Guidelines. Chest 2012;141:e24S-43S.

11. Bates SM, Weitz JI. Coagulation assays. Circulation 2005;112:e53-60

12. Bembea MM, Annich G, Rycus P, et al. Variability in anticoagulation management of patients on extracorporeal membrane oxygenation: an international survey. Pediatr Crit Care Med 2013;14:e77-84.

13. Esper SA, Welsby IJ, Subramaniam K, et al. Adult extracorporeal membrane oxygenation: an international survey of transfusion and anticoagulation techniques. Vox 
Sang 2017;112:443-52.

14. Finley A, Greenberg C. Review article: heparin sensitivity and resistance: management during cardiopulmonary bypass. Anesth Analg 2013;116:1210-22.

15. Despotis GJ, Gravlee G, Filos K, et al. Anticoagulation monitoring during cardiac surgery: a review of current and emerging techniques. Anesthesiology 1999;91:1122-51.

16. Ojito JW, Hannan RL, Burgos MM, et al. Comparison of point-of-care activated clotting time systems utilized in a single pediatric institution. J Extra Corpor Technol 2012;44:15-20.

17. Holbrook A, Schulman S, Witt DM, et al. Evidence-based management of anticoagulant therapy: Antithrombotic Therapy and Prevention of Thrombosis, 9th ed: American College of Chest Physicians Evidence-Based Clinical Practice Guidelines. Chest 2012;141:e152S-84S.

18. Wool GD, Lu CM; Education Committee of the Academy of Clinical Laboratory Physicians and Scientists. Pathology consultation on anticoagulation monitoring: factor X-related assays. Am J Clin Pathol 2013;140:623-34.

19. Atallah S, Liebl M, Fitousis K, et al. Evaluation of the activated clotting time and activated partial thromboplastin time for the monitoring of heparin in adult extracorporeal membrane oxygenation patients. Perfusion 2014;29:456-61.

20. Fitousis K, Klasek R, Mason PE, et al. Evaluation of a pharmacy managed heparin protocol for extracorporeal membrane oxygenation patients. Perfusion 2017;32:238-44.

21. Yie K, Chon SH, Na CY. Activated clotting time test alone is inadequate to optimize therapeutic heparin dosage adjustment during post-cardiopulmonary resuscitational extracorporeal membrane oxygenation (e-CPR). Perfusion 2016;31:307-15.

22. Delmas C, Jacquemin A, Vardon-Bounes F, et al. Anticoagulation Monitoring Under ECMO Support: A Comparative Study Between the Activated Coagulation Time and the Anti-Xa Activity Assay. J Intensive Care Med 2018. [Epub ahead of print].

23. Belk KW, Laposata M, Craver C. A comparison of red blood cell transfusion utilization between anti-activated factor $\mathrm{X}$ and activated partial thromboplastin monitoring in patients receiving unfractionated heparin. J Thromb Haemost 2016;14:2148-57.

24. Schechter T, Finkelstein $Y$, Ali M, et al. Unfractionated heparin dosing in young infants: clinical outcomes in a cohort monitored with anti-factor Xa levels. J Thromb Haemost 2012;10:368-74.

25. Irby K, Swearingen C, Byrnes J, et al. Unfractionated heparin activity measured by anti-factor Xa levels is associated with the need for extracorporeal membrane oxygenation circuit/membrane oxygenator change: a retrospective pediatric study. Pediatr Crit Care Med 2014;15:e175-82.

26. Chu DC, Abu-Samra AG, Baird GL, et al. Quantitative measurement of heparin in comparison with conventional anticoagulation monitoring and the risk of thrombotic events in adults on extracorporeal membrane oxygenation. Intensive Care Med 2015;41:369-70.

27. Northrop MS, Sidonio RF, Phillips SE, et al. The use of an extracorporeal membrane oxygenation anticoagulation laboratory protocol is associated with decreased blood product use, decreased hemorrhagic complications, and increased circuit life. Pediatr Crit Care Med 2015;16:66-74.

28. Niebler RA, Parker H, Hoffman GM. Impact of Anticoagulation and Circuit Technology on Complications During Extracorporeal Membrane Oxygenation. ASAIO J 2018. [Epub ahead of print].

29. Stansfield BK, Wise L, Ham PB 3rd, et al. Outcomes following routine antithrombin III replacement during neonatal extracorporeal membrane oxygenation. J Pediatr Surg 2017;52:609-13.

30. Byrnes JW, Swearingen CJ, Prodhan P, et al. Antithrombin III supplementation on extracorporeal membrane oxygenation: impact on heparin dose and circuit life. ASAIO J 2014;60:57-62.

31. Niebler RA, Christensen M, Berens R, et al. Antithrombin replacement during extracorporeal membrane oxygenation. Artif Organs 2011;35:1024-8.

32. Hans GA, Besser MW. The place of viscoelastic testing in clinical practice. Br J Haematol 2016;173:37-48.

33. Whiting P, Al M, Westwood M, et al. Viscoelastic pointof-care testing to assist with the diagnosis, management and monitoring of haemostasis: a systematic review and cost-effectiveness analysis. Health Technol Assess 2015;19:1-228, v-vi.

34. Bolliger D, Zenklusen U, Tanaka KA. Point-ofcare coagulation management algorithms during ECMO support: are we there yet? Minerva Anestesiol 2016;82:1000-9.

35. Koster A, Faraoni D, Levy JH. Argatroban and Bivalirudin for Perioperative Anticoagulation in Cardiac Surgery. Anesthesiology 2018;128:390-400.

36. Beiderlinden M, Treschan T, Görlinger K, et al. Argatroban in extracorporeal membrane oxygenation. Artif Organs 2007;31:461-5.

37. Menk M, Briem P, Weiss B, et al. Efficacy and safety 
of argatroban in patients with acute respiratory distress syndrome and extracorporeal lung support. Ann Intensive Care 2017;7:82.

38. Sanfilippo F, Asmussen S, Maybauer DM, et al. Bivalirudin for Alternative Anticoagulation in Extracorporeal Membrane Oxygenation: A Systematic Review. J Intensive Care Med 2017;32:312-9.

39. Berei TJ, Lillyblad MP, Wilson KJ, et al. Evaluation of Systemic Heparin Versus Bivalirudin in Adult Patients: Supported by Extracorporeal Membrane Oxygenation. ASAIO J 2018;64:623-9.

40. Warkentin TE, Greinacher A, Craven S, et al. Differences in the clinically effective molar concentrations of four direct thrombin inhibitors explain their variable prothrombin time prolongation. Thromb Haemost 2005;94:958-64.

41. Abdeen MS, Albert A, Maxhera B, et al. Implanting permanent left ventricular assist devices in patients on veno-arterial extracorporeal membrane oxygenation support: do we really need a cardiopulmonary bypass machine? Eur J Cardiothorac Surg 2016;50:542-7.

42. Hoechter DJ, Shen YM, Kammerer T, et al.

Cite this article as: Koster A, Ljajikj E, Faraoni D. Traditional and non-traditional anticoagulation management during extracorporeal membrane oxygenation. Ann Cardiothorac Surg 2019;8(1):129-136. doi: 10.21037/acs.2018.07.03
Extracorporeal Circulation During Lung Transplantation Procedures: A Meta-Analysis. ASAIO J 2017;63:551-61.

43. Ljajikj E, Zitttermann A, Morshuis M, et al. Bivalirudin Anticoagulation for Left Ventricular Assist Device Implantation on an Extracorporeal Life-Support System in Patients with Heparin-Induced Thrombocytopenia Antibodies. Interact Cardiovasc Thorac Surg 2017;25:898-904.

44. Hillebrand J, Sindermann J, Schmidt C, et al. Implantation of left ventricular assist devices under extracorporeal life support in patients with heparin-induced thrombocytopenia. J Artif Organs 2015;18:291-9.

45. ELSO Anticoagulation Guideline. Available online: https://www.elso.org/portals/0/files/ elsoanticoagulationguideline8-2014-table-contents.pdf

46. Baluwala I, Favaloro EJ, Pasalic L. Therapeutic monitoring of unfractionated heparin - trials and tribulations. Expert Rev Hematol 2017;10:595-605.

47. Vandiver JW, Vondracek TG. Antifactor Xa levels versus activated partial thromboplastin time for monitoring unfractionated heparin. Pharmacotherapy 2012;32:546-58. 